\title{
Dosimetric impact of point A definition on high-dose-rate brachytherapy for cervical cancer: evaluations on conventional point $A$ and MRI-guided, conformal plans
}

\author{
James Anderson, PhD, Yunfei Huang, PhD, Yusung Kim, PhD \\ Radiation Oncology Department, University of lowa, USA
}

\begin{abstract}
Purpose: To investigate the dosimetric impact of point A definitions on both conventional point A plans and MRIguided conformal high-dose-rate (HDR) brachytherapy plans.

Material and methods: Fifty-five HDR plans of 36 patients with FIGO stage I to IV cervical cancer were retrospectively studied; these included 30 conventional treatments and 25 conformal plans. Two different point A definitions were explored: the revised Manchester point $A$ and the new point $A$ as recommended by the American Brachytherapy Society. Conventional plans were produced by varying only the point A definition and the normalized isodose lines. Conformal plans were retrospectively generated per GEC-ESTRO recommendations based upon 3.0 Tesla MRI data.

Results: Small yet significant variations were found in point A locations (mean: $0.5 \mathrm{~cm}$, maximum: $2.1 \mathrm{~cm}, p<0.001$ ). The use of a new point A caused minimal dose variation for both conventional and conformal plans. Conventional plans normalized to the new point A generated up to 12\% (avg. 1-3\%) higher overall dose in terms of higher total reference air kerma than plans normalized to other points. Dosimetric changes due to point A definitions were up to 11-12\% (avg. less than $2 \%$ ) on target volumes or organs-at-risk.

Conclusions: For both conventional and conformal plans, the new point A definition leads to smaller variations caused during implant and/or differences in patient anatomy. Using the new point A is expected to produce more consistent brachytherapy plans and improve outcome analysis.
\end{abstract}

Key words: brachytherapy, cervical cancer, high-dose-rate brachytherapy, MRI-guided, point A.

\section{Purpose}

Nearly 12200 new cases of cervical cancer are diagnosed annually in the U.S. [1]. Cervical cancer is a leading health problem worldwide, causing approximately 40000 deaths per year in developed nations and 234000 in developing counties annually. The standard of care for cervical cancer requires concomitant chemotherapy with external-beam radiotherapy (EBRT) and brachytherapy (BT). The BT treatment planning technique, even for high-dose-rate treatments (HDR), has remained unchanged for decades. Utilizing 2-dimensional (2-D) orthogonal radiographs, a plan is created using the Manchester system in which the radiation dose is prescribed to point $\mathrm{A}$ : an estimate of where the uterine artery intersects with the ureter $[2,3]$ irrespective of an individual patient's tumor characteristics. Updates have focused on how point $\mathrm{A}$ is defined [3,4]. The variations of point A (see Fig. 1 of reference [4]) often occurs in a high-gradient region of the isodose distribution. Point A remains the most frequent prescription method $(76 \%)$ in North America [5]. However, studies of on the impact of different definitions of point A are limited to conference abstracts [6-9].

The use of 2-D imaging in HDR BT is shifting towards the use of magnetic resonance imaging (MRI) to acquire volumetric data of the macroscopic tumor region $[10,11]$. MRI guidance allows treatments to be optimized to tumor volumes while sparing organs-at-risk (OARs). Computed tomography (CT), though effective for other treatment sites, is inferior to MRI in accurately distinguishing the tumor and tissues of the cervix [12]. With the advent of 3-dimensional (3-D) imaging the prescription dose shifts away from point A to the clinical target volume (CTV) [5]. Where previously point A would be treated to the prescription dose, the new target for 3-D image guided conformal treatments is for $90 \%$ of the high-risk clinical target volume (HR-CTV $\left.\mathrm{D}_{90}\right)$ to re- 

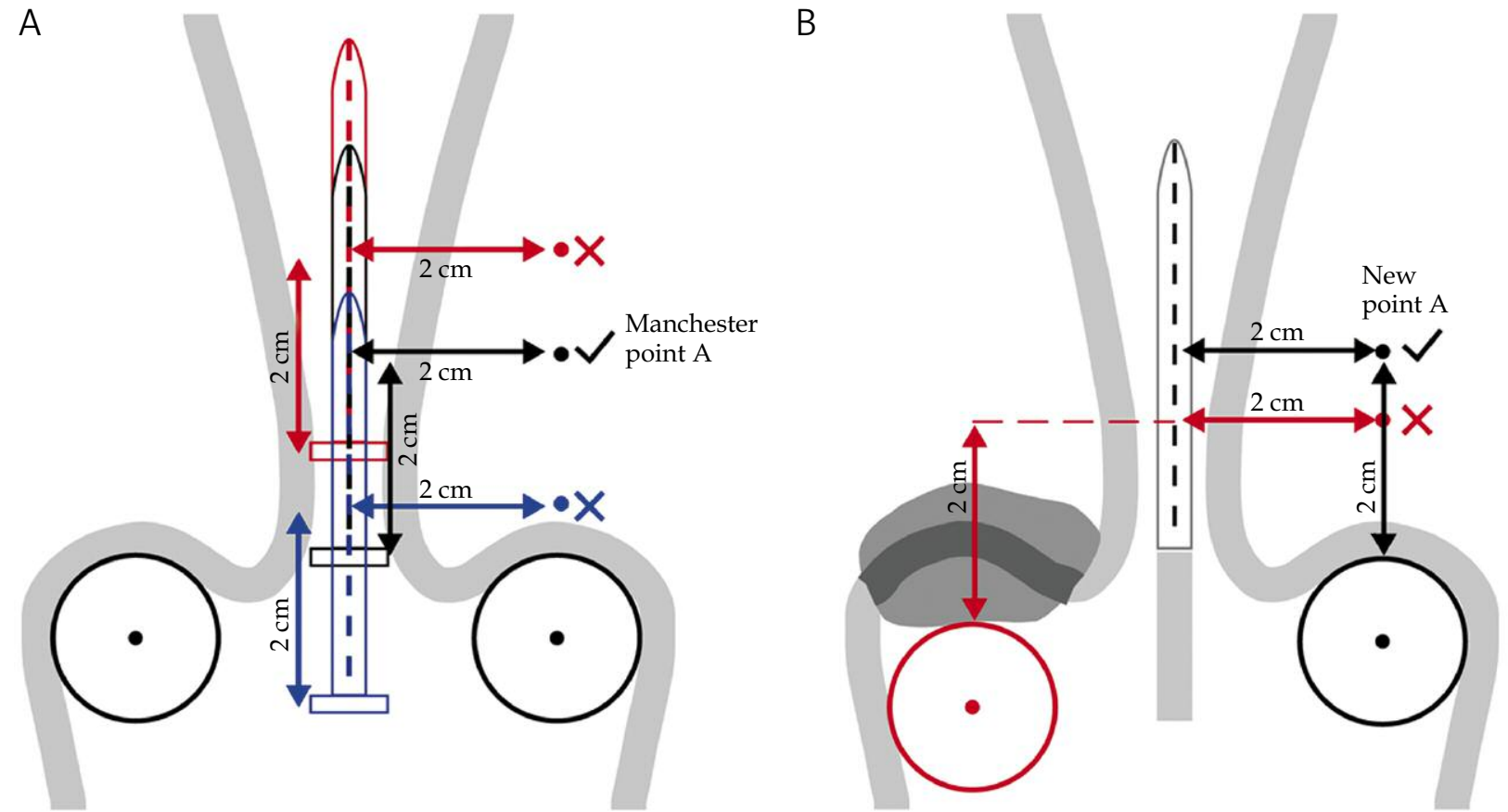

Fig. 1. Definitions and potential errors of Manchester point A and new point A. A) Manchester point A. B) New point A

ceive at least the prescribed dose [13]. The international MRIguided, conformal BT protocol (EMBRACE: An intErnational study on MRI-guided BRachytherapy in locally Advanced CErvical cancer) recommends that HR-CTV $D_{90}$ receives the conventional point A dose. Recent studies have reported significant correlation between dose-volume-histogram (DVH) parameters $\left(\mathrm{D}_{90}\right.$ and $\left.\mathrm{D}_{100}\right)$ and local tumor control. However, the Gynaecological European Group of Curietherapie and the European Society for Therapeutic Radiology and Oncology (GEC-ESTRO) still recommend the recording of conventional point A doses during 3D-image based planning, at least for a transition period [10]. Also recommended is the investigation of correlation back to conventional dose measurements: point A dose and the volumetric-dose in CTV. Both GEC-ESTRO recommendations and the EMBRACE protocol recommend MRI-guided, conformal BT plans report point A doses even when dose is prescribed to HR-CTV D 90 . However, the effect of point A definition on these correlations has not been reported until this present study.

\section{Material and methods}

\section{The definitions of Manchester point $A$ and new point $A$}

The original point A concept of the Manchester System reported in 1938 [2] has never changed: $2 \mathrm{~cm}$ lateral to the central canal of the uterus and $2 \mathrm{~cm}$ up from the mucous membrane of the lateral fornix, in the axis of the uterus. The superior aspects of the vaginal ovoids were originally used as a landmark for the vaginal fornix [2]. In 1953, a cervical flange was proposed due to the difficulty recognizing the ovoids superior edge on radiographs [3], this was referred to as revised point $\mathrm{A}$, although it creates a wider variation of point A locations due to the tandem placement being independent of the ovoid position [4]. To minimize these variations, many institutions developed their own definition of point $\mathrm{A}$, including the use of the superior tip of the ovoids in a sagittal radiograph as a landmark for the vaginal fornix $[8,9]$. In an effort to standardize point A definitions, the American Brachytherapy Society (ABS) introduced a new point A definition [4]. This study compares the following two definitions of point A (Fig. 1):

- Manchester point A: $2 \mathrm{~cm}$ superior to the tandem flange and $2 \mathrm{~cm}$ lateral from the center of tandem. This was the revised point A from 1953 [3].

- New point A: The new point definition as recommended by the ABS. Draw a line through the center of each ovoid. $(R+2) c m$ superiorly along the tandem from the intersection of this line, where $R$ is the radius of the ovoid and $2 \mathrm{~cm}$ lateral from the center of the tandem.

It should be noted that all point A definitions will be co-located when the applicator is correctly implanted. For clarity, this work will use the form "Manchester point A" or "new point $A$ " to refer to the specific definitions and "point $\mathrm{A}$ " to refer to the general treatment concept.

\section{HDR treatment plans}

A total of $55 \mathrm{HDR}$ plans from patients with biopsy-proven cervical cancer who received radiotherapy (RT) were retrospectively studied after approval from the local Institutional Review Board. All 55 plans were clinically generated per conventional, point A based techniques. Thirty of these plans were guided by 2-D orthogonal radiographs (Fig. 2A) while the other 25 used MRI datasets (Fig. 2 D-F) from a high resolution (3.0 Tesla) Siemens MAGNETOM Trio 3T MR scanner (Siemens Medical Solutions, Erlangen, Germany ${ }^{\circledR}$ ). Only the 25 plans using MRI were analyzed 

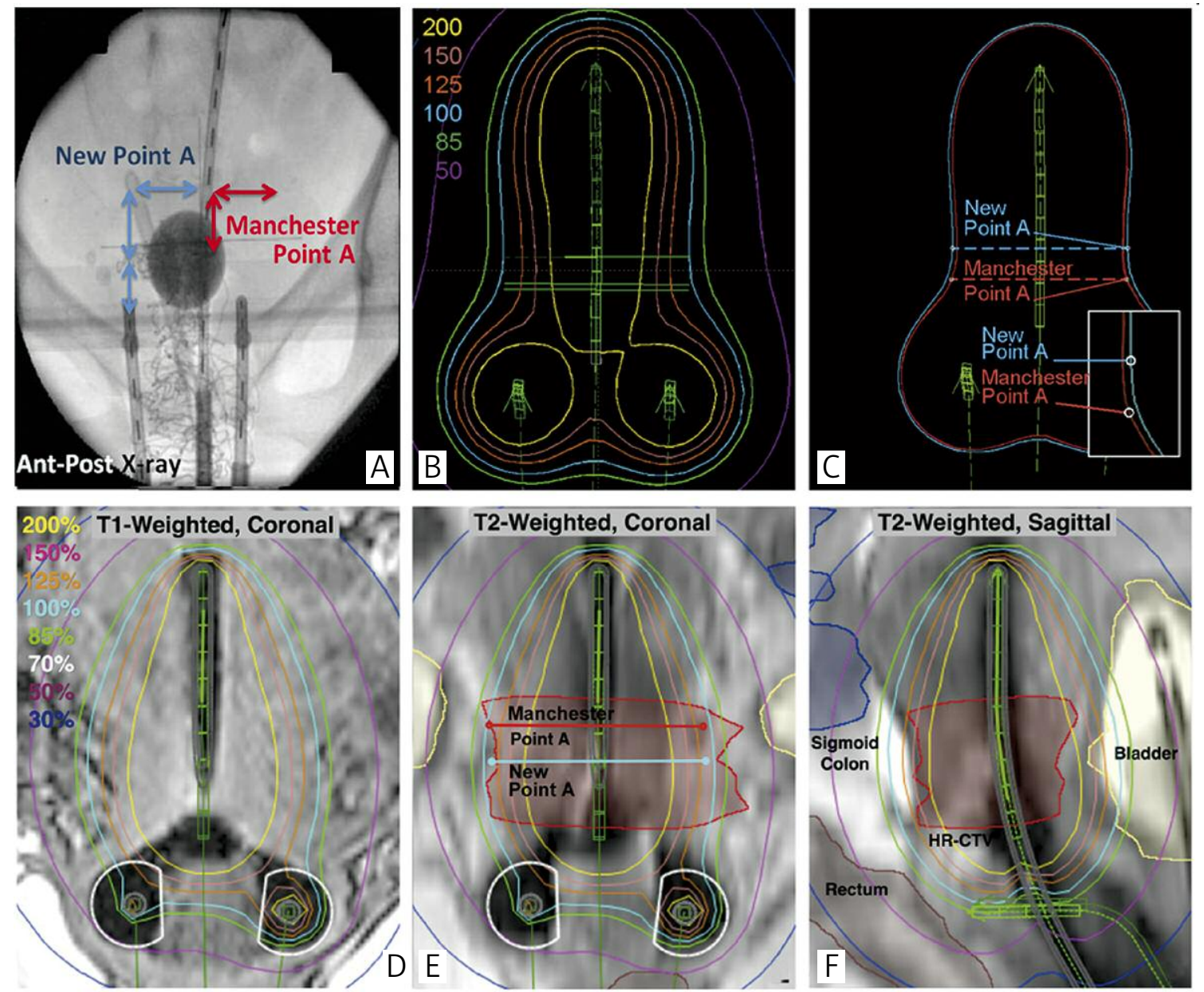

Fig. 2. Part (A) shows coronal and sagittal radiograph images and example locations of the two points, (B) shows the full isodose set for a normalized to new point A, and (C) shows the $100 \%$ isodose lines for both point A definitions. Parts (D) through (F) show high resolution (3.0 Tesla) MR images with HR-CTV and OAR contours and conformal plan isodose lines

using DVH parameters. In addition, the 25 plans were retrospectively re-planned using conformal planning techniques. In the authors' institutional protocol, the majority of patients with FIGO (International Federation of Gynecology and Obstetrics) stage Ib2 and above cervical cancer received EBRT, HDR BT and weekly concomitant cisplatin infusion. BT fractionation schemes consisted of either 3 fractions of 7.5 Gy or 5 fractions of $5.5 \mathrm{~Gy}$, with occasional minor variations. A titanium Fletcher-Suit-Declos style tandem-and-ovoids (T\&O) applicator (Varian Medical Systems, Palo Alto, $\left.\mathrm{CA}^{\circledR}\right)$ was used for all plans, along with the BrachyVision ${ }^{\mathrm{TM}}$ treatment planning system (version 8.9, Varian Medical Systems, Palo Alto, $\left.\mathrm{CA}^{\circledR}\right)$.

All conventional, clinical plans had been optimized to generate standard pear-shaped isodose distributions using reference lines that were located $2 \mathrm{~cm}$ lateral from the tandem and $0.5 \mathrm{~cm}$ lateral from each ovoid's surface (Fig. 2) [4]. The locations of Manchester point A and new point A were retrospectively defined by a single person to avoid inter-physicist variation. For both point definitions, the stand pear-shaped isodose lines were re-normalized such that the point received the prescribed dose. No changes were made to the loading pattern.
The 25 plans using MRI were also retrospectively replanned with the intention of generating MRI-guided, conformal plans (Fig. 2 D-F) in accordance with GEC-ESTRO recommendations $[10,11]$ and EMBRACE protocol. The details of the MRI scan protocols for the T\&O HDR BT procedure were summarized in the previous study [14]. An experienced physician contoured the intermediate risk CTV (IR-CTV), the high risk CTV (HR-CTV) and organs-at-risk (OAR) according to GEC-ESTRO recommendations [10,11] and EMBRACE protocol. A conformal plan was optimized to achieve the following: HR-CTV $D_{90}$ was to receive the prescription dose and $D_{2 c c}$ (the minimum dose within the 2 cc of tissue receiving the greatest dose) of each OAR was to receive less than the limits of the GEC-ESTRO recommendations [10,11] and EMBRACE protocol. The recommended limits of $\mathrm{D}_{2 \mathrm{cc}}$ for the rectum, bladder and sigmoid colon are $75 \mathrm{~Gy}, 90 \mathrm{~Gy}$, and $75 \mathrm{~Gy}$, respectively. These limits are the combined EBRT and HDR doses in terms of EQD2 (equivalent dose in $2 \mathrm{~Gy}$ fractions of EBRT, using an $\alpha / \beta$ value of 3). EBRT plan data were used during conformal HDR plan optimization procedures to apply the recommended OAR limits. The hybrid-inverse optimization described in our previous work [15] was utilized for all con- 
formal plans (Fig. 2): starting with a conventional point A plan, dose-volume objectives were set and inverse planning performed. The isodose lines and DVH measurements of the optimized plan were then checked and further inverse planning or graphical dose shaping used where necessary.

\section{Analysis}

Among total 55 conventional plans, the 30 plans guided by 2-D radiographs were analyzed by measuring the volume receiving $100 \%$ or more of the prescription dose $\left(V_{100 \%}\right)$, the total reference air kerma (TRAK), and the dose to the rec-

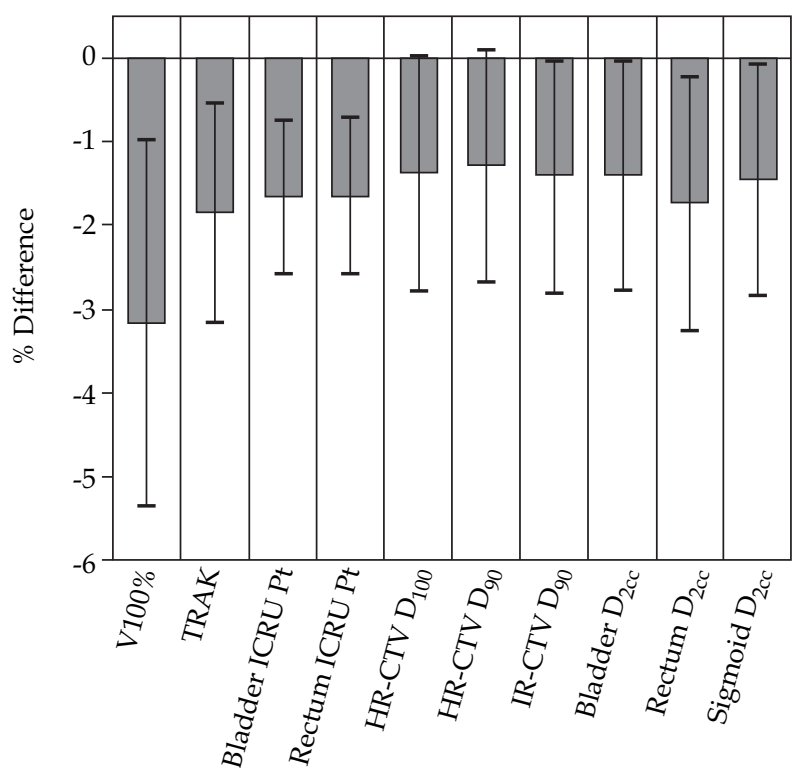

Fig. 3. Average percent differences of Manchester point A plans when normalized to new point A plans. Negative values indicate lower results for the Manchester point A plans. The 95\% confidence interval is marked

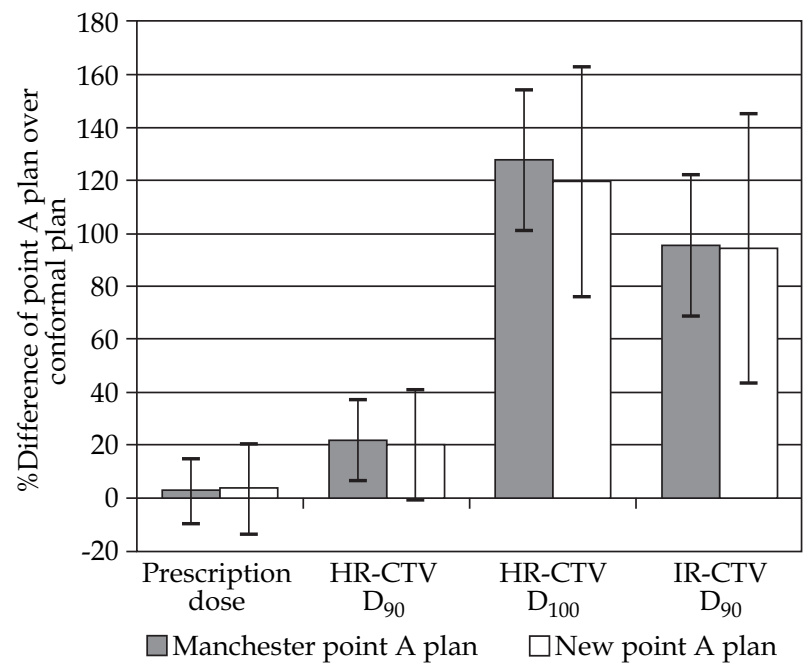

Fig. 4. Percent differences between Manchester point A (point $\mathrm{H}$ ) doses and prescription doses \& tumor coverage metric from MRI-guided, conformal plans. Positive values indicate higher point doses. The $95 \%$ confidence interval is marked tum and bladder points of ICRU report \#38 (International Commission on Radiation Units and Measurement). The other 25 conventional plans using MRI were further analyzed using DVH parameters, including the minimum dose received by $90 \%$ of the HR-CTV $\left(D_{90}\right)$ and the minimum dose to the hottest $2 \mathrm{cc}$ of each OAR $\left(\mathrm{D}_{2 \mathrm{cc}}\right)$. All DVH parameters were analyzed only for HDR plans no EBRT plan data were analyzed. For each measurement of a plan renormalized using Manchester point A (Manchester point A plan), for example, a corresponding measurement was taken using the new point A normalized plan (new point $\mathrm{H}$ plan). As the focus was on systematic differences between these plans, we took the percent difference of each pair as the first step of our analysis. This was done with respect to the new point $A$ plans such that negative results indicated a lower value in the point A plan (see Fig. 3).

The 25 conformal plans using MRI were also analyzed by DVH parameters, along with the 2-D parameters $\mathrm{V}_{100 \%}$, TRAK, and rectal and bladder point doses. In conformal plans, the correlation between tumor coverage values such as HR-CTV $\mathrm{D}_{90}, \mathrm{D}_{100}$ or IR-CTV $\mathrm{D}_{90}$ and point $\mathrm{A}$ doses are of interest, since the majority of clinical outcomes are linked with these point measurements. It is noted that conformal plans do not use point A to generate plans, but use HR-CTV and OAR to perform volume optimization. Therefore, we quantified how the correlation is affected by different point A definitions for a given conformal plan. Specifically percent differences between Manchester point $A$ or new point A dose and tumor coverage values $\left(D_{90}\right.$ or $\left.D_{100}\right)$ were taken with respect to the dose volume measurement values, with negative results indicating a lower value in the point dose (cf. Fig. 4). For all dose-volume parameter analysis (i.e. $\mathrm{D}_{90}, \mathrm{D}_{100}$, and $\mathrm{D}_{2 \mathrm{cc}}$ ), physical doses were converted to EQD2 values using $\alpha / \beta=3$ for healthy tissue and $\alpha / \beta=10$ for target volumes $[10,11]$.

\section{Results}

We found that a conventional, point A based plan is minimally affected by variations caused by implant procedure and/or patient anatomy when new point A definition was used. For any plan, there is both a left and right side point $A$ and the normalization ensures that their average dose values equal the prescribed dose. We observed that the percent deviations of the higher single point A values with respect to the prescription doses were up to $9.5 \%$ (mean 1.7\%, $\mathrm{CI}_{95} \pm 0.5 \%, p<0.001$ ) and for new point $\mathrm{A}$ up to $6.7 \%$ (mean $\left.1.5 \%, \mathrm{Cl}_{95} \pm 0.3 \%, p<0.001\right)$. Across the 55 plans, Manchester point $\mathrm{A}$ was found to be mean distance of $0.6 \mathrm{~cm}$ from new point $\mathrm{A}\left(\mathrm{CI}_{95} 0.1 \mathrm{~cm}, \max 2.1 \mathrm{~cm}, p<0.001\right)$. In other words, a small yet significant variation was found in point A location.

Figure 2A shows a sample radiograph of a case with a distance of over $2 \mathrm{~cm}$ between different point A definitions, which caused the plotted separation in $100 \%$ isodose lines. The dosimetric impact of point A definition is shown in Fig. 3: we plotted the average percent difference of measurements from plans normalized to point $\mathrm{A}$ with respect to corresponding measurements from those normalized to new point A. A significant $(p<0.005)$ difference was seen between different point $A$ definitions when $V_{100 \%}$, TRAK and ICRU 
rectal and bladder point doses were considered. These differences were all less than $2 \%$. The biggest dosimetric impact due to point $A$ definitions were in the $V_{100 \%}$ and TRAK values, both of which represent the overall amount of radiation delivered. The maximum differences between Manchester point $\mathrm{A}$ and new point $\mathrm{A}$ were $20 \%$ and $12 \%$ for $\mathrm{V}_{100 \%}$ and TRAK, respectively. We found that plans normalized to point $A$ had lower dosimetric values, on average, than those normalized to new point A (Fig. 3). This is because new point A was generally located superiorly to Manchester point A, resulting in a larger area enclosed by the $100 \%$ isodose line for new point A plans (Fig. 2). The right side of Fig. 3 shows the mean percent differences for each CTV and OAR in which all variations were less than $2 \%$.

We next considered MRI-guided, conformal plans. In each of these plans, the point dose values at Manchester point $\mathrm{A}$ and new point $\mathrm{A}$ were recorded and compared to the prescription dose and volumetric tumor coverage values (HR-CTV $\mathrm{D}_{90}, \mathrm{D}_{100}$ and IR-CTV $\mathrm{D}_{90}$ ) (see Fig. 4). For the comparison with EQD2 values, all point A doses and prescription doses were converted to EQD2 values using $\alpha / \beta=10[10,11]$. Figure 4 shows the percent differences of point A doses, the prescription dose and tumor coverage values. Positive values indicate higher point doses. The relationship between point A doses and volumetric tumor coverage values were not considerably changed due to point A definition. The mean changes of no more than $2 \%$ were observed on the prescription dose, HR-CTV $D_{90}$ and IR-CTV $\mathrm{D}_{90}$ due the point A definition. The relationship between point $A$ dose and HR-CTV $D_{100}$ was maximally changed (i.e. mean 9\%) due to the point A definition. New point A still showed smaller variations from the prescribed dose than those of Manchester point A.

\section{Discussion}

The variance seen between point A definitions comes from two main sources: i) imprecise implantation of the applicator and/or ii) inter-planner differences while defining either point $A$ on 2-D radiographs or 3-D imaging datasets. The latter should be limited if all planners are well trained and has been removed from this study entirely as the same investigator performed the task in all cases. There was no such restriction, however, on the applicator implantation: this process is inherently imprecise due to visualization difficulties and patient anatomy and is the source of the deviation between point $A$ definitions. The most recent ABS guideline 2012 [16] updated the new point A definition to first move to the midpoint between the two ovoids, then superiorly $\mathrm{R}+2 \mathrm{~cm}$ along the tandem (see Fig. 1 in Ref. [16]). Following the updated definition is expected to result in a yet more robust point definition.

In this study, mean deviations were found to have a minimal effect on conventional plans, as only small differences were found between plans normalized to different point definitions. This finding matches the study of Eng et al. [6] showing less than $3 \%$ variation between Manchester point $A$ and new point $A$ plans when considering doses to point $B$, ICRU rectum point or ICRU bladder point. Point $B$ was reported as a poor surrogate for pelvic lymph node dose [17], so it was excluded as a dosimetric measurement in this study.

We found the plans normalized to new point $A$ had on average $2-3 \%$ higher TRAK or $\mathrm{V}_{100 \%}$ values over those normalized to Manchester point A. These findings match the results of Howell et al. [7], showing that a plan normalized to new point A generated slightly higher doses. There is no literature describing the impact on clinical outcomes due to a $2-3 \%$ increase in overall dose. The variation in tumor coverage for conventional point A plans was summarized in our previous study [18]. Here, the mean variations due to the use of different point A definition were found to be relatively small. However, we found maximal TRAK changes of $11-12 \%$ and volumetric metrics such as HR-CTV $\mathrm{D}_{90}$. Thus, the correlation between point $\mathrm{A}$ doses and volumetric tumor coverage (e.g. HR-CTV $D_{90}$ ) can be changed up to $11-12 \%$ due to different point A definitions. It is recommended that the use of a new point A definition for those studies is adopted. With the development of MRIguided, conformal BT, the correlation between conventional point A doses and dose-volume parameters of HR-CTV/ IR-CTV have been reported [13], although no phase III clinical outcome studies between MRI-guided, conformal BT and conventional point A based BT are presently available. Using the more stable new point A definition is expected to enable better clinical outcome analysis, including deeper understanding of the correlations between point A dose and dose-volume parameters of the HR-CTV.

\section{Acknowledgments}

This work was supported in part by a research collaboration grant from Varian. The authors want to express their gratitude to Jacobson Geraldine, Bhatia Sudershan, and Wenqing Sun for their contouring.

\section{References}

1. American-Cancer-Society. Cancer Facts \& Figures 2010; available at: http:/ /www.cancer.org/acs/groups/content/@nho/ documents/document/acspc-024113.pdf.

2. Tod M, Meredith W. A dosage system for use in the treatment of cancer of the uterine cervix. Br J Radiol 1938; 11: 809-824.

3. Tod M, Meredith WJ. Treatment of cancer of the cervix uteri, a revised Manchester method. Br J Radiol 1953; 26: 252-257.

4. Nag S, Erickson B, Thomadsen B et al. The American Brachytherapy Society recommendations for high-dose-rate brachytherapy for carcinoma of the cervix. Int J Radiat Oncol Biol Phys 2000; 48: 201-211.

5. Viswanathan AN, Erickson BA. Three-dimensional imaging in gynecologic brachytherapy: a survey of the American Brachytherapy Society. Int J Radiat Oncol Biol Phys 2010; 76: 104-109.

6. Eng TY, Cummins S, Baake D. Point A or Point H in Prescribing High-Dose-Rate (HDR) Intracavitary Brachytherapy for Cervical Carcinoma? Int J Radiat Oncol Biol Phys 2007; 69: S396S397.

7. Howell RM, Prete JJ, Wiatrowski WA et al. Calculation/comparison of dose to point " $\mathrm{H}$ " according to the ABS recommendations for HDR brachytherapy for carcinoma of the cervix to the dose to point " $\mathrm{A}$ " based on the revised Manchester system. Int J Radiat Oncol Biol Phys 2001; 51: 330-331.

8. Kim Y, Huang Y, Bayouth JE et al. Dosimetric consequences of the prescription point $\mathrm{H}$ of ABS recommendation in the era of MRI guided brachytherapy for cervical cancer: based on 
GYN GEC-ESTRO recommendations of MRI guided brachytherapy. Int J Radiat Oncol Biol Phys 2008; 72: S586-587.

9. Huang Y, Kim Y. On the Impact of Prescription Point A on HRCTV in MRI-Guided HDR Brachytherapy for Cervical Cancer. Int J Radiat Oncol Biol Phys 2010; 78: S424.

10. Potter R, Haie-Meder C, Van Limbergen E et al. Recommendations from gynaecological (GYN) GEC ESTRO working group (II): concepts and terms in 3D image-based treatment planning in cervix cancer brachytherapy-3D dose volume parameters and aspects of 3D image-based anatomy, radiation physics, radiobiology. Radiother Oncol 2006; 78: 67-77.

11. Haie-Meder C, Potter R, Van Limbergen E et al. Recommendations from Gynaecological (GYN) GEC-ESTRO Working Group (I): concepts and terms in 3D image based 3D treatment planning in cervix cancer brachytherapy with emphasis on MRI assessment of GTV and CTV. Radiother Oncol 2005; 74: 235-245.

12. Viswanathan AN, Dimopoulos J, Kirisits $C$ et al. Computed tomography versus magnetic resonance imaging-based contouring in cervical cancer brachytherapy: results of a prospective trial and preliminary guidelines for standardized contours. Int J Radiat Oncol Biol Phys 2007; 68: 491-498.

13. Kirisits $C$, Potter R, Lang $S$ et al. Dose and volume parameters for MRI-based treatment planning in intracavitary brachytherapy for cervical cancer. Int J Radiat Oncol Biol Phys 2005; 62: 901-911.

14. Kim Y, Muruganandham M, Modrick JM et al. Evaluation of artifacts and distortions of titanium applicators on 3.0-Tesla MRI: feasibility of titanium applicators in MRI-guided brachytherapy for gynecological cancer. Int J Radiat Oncol Biol Phys 2011; 80: 947-955.

15. Kim Y, Modrick JM, Bayouth JE et al. Logistics of tandem and ovoids HDR plan optimization in MRI-guided brachytherapy for cervical cancer: comparisons of standard, graphical, and inverse optimization. Brachytherapy 2009; 8: 121-122

16. Viswanathan AN, Thomadsen B. American Brachytherapy Society consensus guidelines for locally advanced carcinoma of the cervix. Part I: general principles. Brachytherapy 2012; 11: 33-46.

17. Lee LJ, Sadow CA, Russell A et al. Correlation of point B and lymph node dose in 3D-planned high-dose-rate cervical cancer brachytherapy. Int J Radiat Oncol Biol Phys 2009; 75: 803-809.

18. Kim Y, Xia J, Sun W et al. Tandem and ovoids brachytherapy for cervical cancer when evaluated by high resolution (3.0 Tesla) MRI. Med Phys 2010; 37: 3348. 